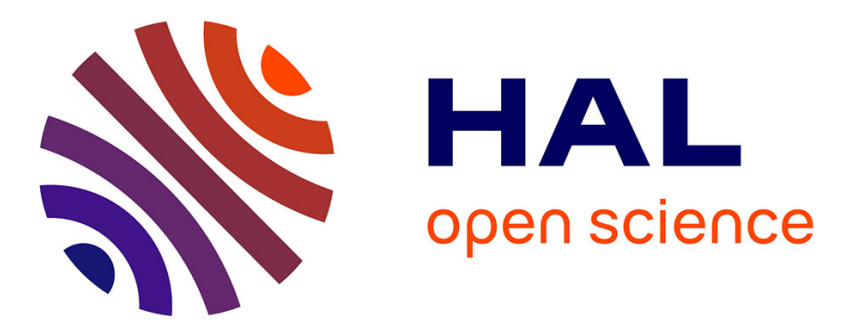

\title{
Les débuts de l'informatique dans les universités: un moment de la différenciation géographique des pôles scientifiques français.
}

Michel Grossetti, Pierre-Eric Mounier-Kuhn

\section{- To cite this version:}

Michel Grossetti, Pierre-Eric Mounier-Kuhn. Les débuts de l'informatique dans les universités: un moment de la différenciation géographique des pôles scientifiques français.. Revue française de sociologie, 1995, 36 (2), pp.295-324. halshs-00476924

\section{HAL Id: halshs-00476924 \\ https://shs.hal.science/halshs-00476924}

Submitted on 27 Apr 2010

HAL is a multi-disciplinary open access archive for the deposit and dissemination of scientific research documents, whether they are published or not. The documents may come from teaching and research institutions in France or abroad, or from public or private research centers.
L'archive ouverte pluridisciplinaire HAL, est destinée au dépôt et à la diffusion de documents scientifiques de niveau recherche, publiés ou non, émanant des établissements d'enseignement et de recherche français ou étrangers, des laboratoires publics ou privés. 
Michel Grossetti

Centre Interdisciplinaire d'Études Urbaines

(CIEU-CNRS UA 1146)

et Centre de Recherches Sociologiques

Université de Toulouse-le-Mirail

5 allées A. Machado

31058 Toulouse Cedex

Tel : 61504301 Fax : 61504209

INTERNET : RGROS@CICT.FR
P. Mounier-Kuhn

Centre de Recherches

Roland-Mousnier

Histoire et Civilisation

CNRS et Univ. Paris-Sorbonne

1 , rue Victor Cousin

75230 Paris Cedex 05

Tel : 46334986 Fax : 40273192

\title{
LES DEBUTS DE L'INFORMATIQUE DANS LES UNIVERSITES
}

\section{UN MOMENT DE LA DIFFERENTIATION GEOGRAPHIQUE DES POLES SCIENTIFIQUES FRANÇAIS.}

\author{
Revue Française de Sociologie XXXVI, n², pp.295-324
}

\begin{abstract}
Résumé
L'histoire des débuts de l'informatique dans les universités françaises permet d'analyser à la fois l'effet des configurations locales sur l'adoption d'une nouvelle discipline et le processus de différenciation des pôles scientifiques. On montre que l'informatique trouve des conditions favorables à son développement comme domaine de recherche là où préexistent des équipes de calcul numérique animées par un professeur/autour d'une chaire. Ces équipes sont présentes dans toutes les facultés des sciences dotées d'écoles d'ingénieurs en électricité, à cause des besoins de ces écoles en enseignement de mathématiques appliquées et de leur situation institutionnelle particulière - elles n'ont aucune autonomie de recrutement par rapport aux facultés. En rappelant l'origine de ces écoles à Grenoble, Toulouse et Nancy, on esquisse une périodisation du processus de différenciation des pôles scientifiques français en ce qui concerne les sciences appliquées.
\end{abstract}

L'intégration de plus en plus importante de la recherche publique au processus d'innovation technique (Callon, Larédo, Mustar, 1994) et la tendance de celui-ci à se déployer au sein de systèmes locaux associant entreprises, laboratoires et instituts de formation, invite à interroger la territorialisation des institutions scientifiques ellesmêmes. En effet, quel que soit le statut des universités ou des centres de recherche dans la constitution et le fonctionnement des zones d'innovation, la question des disparités géographiques du système scientifique se trouve posée, puisque les sites fortement dotés d'établissements d'enseignement supérieur et de recherche bénéficient potentiellement d'avantages significatifs dans le développement d'activités de haute technologie.

Nous ne disposons que de peu de travaux concernant la géographie des institutions scientifiques, les rares qui existent se présentant comme de simples catalogues (Mailfert, 1991) ou se focalisant essentiellement sur les déséquilibres traditionnels entre Paris et la Province (Brocard, 1991). Or, la répartition sur le territoire national des institutions scientifiques ne se résume pas à la seule opposition Paris/Province, qui doit d'ailleurs être relativisée dans le domaine des sciences appliquées : la région parisienne, qui concentre $50 \%$ des chercheurs CNRS, ne rassemble que $40 \%$ du département Science de la Chimie et $35 \%$ du département Sciences pour l'Ingénieur. Les unités parisiennes ne réalisent que le quart des contrats avec l'industrie, soit autant que celles de la région Rhône-Alpes qui ne représentent que 10\% des effectifs. Pour avoir une valeur explicative, une géographie des institutions scientifiques doit dépasser les grands agrégats statistiques et entrer dans le détail des disciplines et domaines de recherche. On découvre ainsi que la majeure partie du potentiel français de recherche en "Informatique, automatique et traitement du signal" (section 7 du CNRS, l'une des plus "appliquées"), se distribue surtout entre Paris (36\%), Toulouse (25\%) et Grenoble (22\%), ou que $50 \%$ du potentiel de recherche en génie des procédés est concentré à Nancy et Toulouse (Gaillard, 1991).

En approfondissant une analyse de ce type on constate l'existence de pôles scientifiques ayant chacun sa spécificité et son identité. Ces spécificités et identités sont largement le produit de processus historiques qu'il est nécessaire d'interroger pour comprendre la situation actuelle. Les organismes technologiques d'Etat (CEA, INRA, INRIA, etc.), obéissent pour l'implantation de leurs établissements aux politiques publiques d'aménagement du territoire dont l'analyse relève du domaine de la sociologie de la décision (Sfez, 1976), mais la répartition géographique des institutions scientifiques non spécialisées - universités et CNRS - est le produit de processus 
plus complexes où les dimensions scientifiques, organisationnelles et individuelles sont étroitement imbriquées pour produire des effets de continuité et de renforcement progressif des disparités.

Au-delà du renouvellement plus ou moins rapide des unités de recherche, les orientations générales des pôles scientifiques sont marqués par une remarquable continuité. Si Nancy et Toulouse ont autant d'importance pour le génie des procédés, c'est en partie parce que là ont été créés les premiers enseignements de ce domaine en 19491950. Si tant de chercheurs en informatique se trouvent à Grenoble (environ 13\% des effectifs CNRS de la discipline ${ }^{1}$ ), c'est certainement parce que l'IMAG (Institut de Mathématiques Appliquées de Grenoble) fut l'un des premiers laboratoires à se consacrer au calcul numérique dans les années 1950. Si le Laboratoire d'Automatique et d'Analyse des Systèmes (LAAS), créé en 1967 à Toulouse, est le plus important du CNRS, c'est en grande partie parce qu'il s'est constitué sur la base du Laboratoire de Génie Électrique qui fut en 1955 l'un des premiers à travailler sur les servomécanismes.

Les continuités entre la période d'émergence de certaines disciplines appliquées (informatique, automatique, génie des procédés, etc.) et la situation actuelle des pôles scientifiques nous incitent, pour comprendre la constitution de ces pôles, à analyser ce qui s'est joué au moment de l'apparition des disciplines qui en font la spécificité. Ainsi, on peut se demander pourquoi les Facultés des Sciences de Grenoble et Toulouse ont vu naître dès 1956/1957 des équipes de recherche en informatique alors que d'autres comme Montpellier ou Strasbourg en sont dépourvues avant les années quatre-vingt.

Le constat de ces différences considérables de précocité forme le point de départ des travaux sur lesquels s'appuie ce texte ${ }^{2}$, travaux qui s'inscrivent dans un projet général d'étude des processus de différenciation des pôles scientifiques français ${ }^{3}$. L'analyse présentée ici se centre sur une période particulière - de l'après-guerre à 1968 - et les débuts d'une discipline - l'informatique, ce qui permet d'étudier le jeu des configurations locales dans l'introduction de nouveaux domaines de recherche.

Soit le système français d'enseignement supérieur et de recherche à un moment donné, dans un état de structuration institutionnelle et territoriale donné. Arrive de l'extérieur du système une innovation technique majeure, l'une des plus importantes du siècle : l'ordinateur (les premiers prototypes entrent en fonctionnement à la fin des années quarante dans les pays anglo-saxons, les premières installations en France datent de 1955). Comment le système réagit-il ? Que se passe-t-il dans les différents pôles ? Quelles sont les logiques sociales (scientifiques, organisationnelles, individuelles, locales ou générales) qui se révèlent dans ce processus?

Ces questions s'inscrivent dans le cadre d'une sociologie des institutions ${ }^{4}$ scientifiques, qui se différencie d'une sociologie de la science centrée sur les découvertes ou les controverses (Collins, 1982, Farley et Geison, 1982, Latour, 1989) et prend pour objet principal les organisations qui les produisent. Dans la mesure où ces organisations résultent de processus historiques relativement longs, il est intéressant de les aborder non plus seulement en tant que lieux d'activités et d'interactions (Latour et Woolgar, 1979, Lynch, 1985, Knorr-Cetina, 1983) mais aussi du point de vue de leur genèse et de leur évolution en intégrant la dimension de leurs orientations scientifiques. Une telle approche retrouve d'un certain point de vue des préoccupations anciennes de la sociologie de la science (Merton, 1973, Ben David, 1968), mais en les inscrivant dans un tout autre contexte théorique et épistémologique. Si le choix d'un objet comme celui des institutions implique évidemment une posture relativement "externaliste" et l'usage de catégories générales de la sociologie, il ne s'associe ici nullement à un postulat néo-positiviste sur l'activité scientifique et tente d'en intégrer certains éléments internes lorsqu'ils améliorent les systèmes explicatifs. Quant à la préoccupation historique, elle ne vise pas à trouver dans le passé des situations pouvant illustrer un modèle théorique général, mais s'intègre plutôt à une préoccupation de type généalogique cherchant dans quelle mesure la situation actuelle des institutions scientifiques peut s'expliquer, au moins en partie par les conditions de leur genèse.

Si nous considérons l'ensemble des institutions scientifiques et des acteurs qui les animent présents dans une agglomération, nous obtenons un système d'action local, structuré par des enjeux et une histoire commune, que

${ }^{1}$ Comptage réalisé par les auteurs, sur la base de l'annuaire du CNRS, par un tri des unités de la section 7 du Comité national de la recherche scientifique.

2 Un premier travail, soutenu par le Programme Interdisciplinaire de Recherche sur les Technologies, l'Emploi et les Modes de Vie (PIRTTEM) du CNRS, a consisté en une comparaison de trois sites - Grenoble, Toulouse et Nancy - pour l'émergence de trois disciplines - le génie chimique, l'automatique et l'informatique - et a donné lieu à un rapport (Grossetti, 1991). Une recherche plus approfondie centrée sur l'informatique a été menée sur les principaux pôles français. Des séries d'entretiens ont été réalisées à Paris, Strasbourg, Lille, Montpellier et Bordeaux, Rennes-Nantes, Lyon et Marseille. Un travail de dépouillement d'archives (locales et nationales) a complété les entretiens.

3 Pour une présentation synthétique de ces processus, voir (Grossetti, 1994a).

4 Institution est pris ici dans le sens restreint d'organisation non marchande. 
nous pouvons baptiser système scientifique local. L'hypothèse que nous chercherons à argumenter au cours de cette analyse est que la structure institutionnelle et les rapports entre acteurs internes au système, tels qu'ils existent à un moment donné, peuvent expliquer les orientations prises par les institutions scientifiques locales. En particulier, parmi les diverses configurations existant dans les universités françaises de l'après-guerre, celle qui associe une faculté des sciences et des écoles d'ingénieurs se révèle propice au développement des sciences appliquées.

Avant d'examiner ce qui s'est passé dans les onze universités étudiées, il est nécessaire de situer le contexte général dans lequel le développement de l'informatique universitaire prend place. En s'intéressant aux ordinateurs, les universitaires s'inscrivent dans un mouvement plus général. En effet, la diffusion de techniques et de compétences, consécutive à l'installation des nouvelles machines, s'accompagne de la naissance d'une profession et d'une spécialité scientifique — peu importe que le mot "informaticien" n'existe pas encore. En 1955, la France ne compte que de rares spécialistes en programmation, en circuits et en architecture de machines, essentiellement chez les constructeurs, ainsi que dans les équipes universitaires mentionnées ci-dessus. Deux ou trois ans plus tard un véritable milieu professionnel s'est organisé, avec ses associations (Association Française de CalculAFCAL, Association Française de Régulation et d'Automatique-AFRA), ses centres de rencontre ou de propagande (Syndicat national de l'automation, Centre national de l'automatisation), ses manifestations scientifiques et commerciales, ses moyens d'information (revues Chiffres, Automatisme, Automation, Électrocalcul un peu plus tard) auxquels s'ajoutent bientôt les premiers livres sérieux écrits par des Français sur la question $^{5}$. En 1958 est créée la première société de service en informatique, la SEMA. La vitalité de l'informatique française, le départ une fois pris, est attestée par le fait que, au début des années 1960, une dizaine d'entreprises construisent des ordinateurs.

Au sein du système universitaire, l'émergence d'une nouvelle discipline peut toujours s'analyser sous deux aspects : celui de la recherche (apparition du thème au sein d'équipes existantes, création de nouvelles équipes) et celui de l'enseignement (mise en place de cours à différents niveaux des cursus, reconnaissance officielle de la discipline). Dans le cas de l'informatique, il existe un troisième aspect, celui de l'outil technique au service des autres disciplines. D'une façon générale, les calculateurs arrivent les premiers : sans eux, impossible d'enseigner ou de faire de la recherche en informatique. Les enseignements suivent avec plus ou moins de décalage : ils peuvent être assurés par des intervenants extérieurs ou des spécialistes d'autres disciplines. La recherche vient en dernier et s'accompagne toujours d'enseignements, au moins de troisième cycle. L'analyse des débuts de l'informatique dans les différentes universités prend pour point de repère commun l'acquisition de calculateurs.

L'introduction de l'informatique dans les universités s'effectue selon des logiques que l'on peut, en ce qui concerne la recherche, classer en deux grands types :

- les logiques endogènes : des équipes existantes lancent des recherches sur les ordinateurs et la programmation, et finissent par constituer de grands laboratoires, voire de nouveaux instituts,

- les logiques exogènes : l'informatique ne se développe que grâce à l'apport d'enseignants issus des pôles précurseurs.

Ces deux logiques seront le sujet des deux premières parties de l'article. Une troisième partie éclairera les relations causales entre le développement d'écoles d'ingénieurs depuis le début du siècle dans certaines universités, et le démarrage de l'informatique pendant les “Trente Glorieuses".

\section{1 - Logiques endogènes : l'importance des mathématiques appliquées}

Dans les universités françaises, la recherche en informatique naît au sein des mathématiques appliquées et plus précisément du calcul numérique. Le calcul numérique est alors une branche renaissante des mathématiques, qui s'efforce d'acquérir, grâce à un travail de théorisation, un statut lui conférant une plus grande légitimité aux yeux des mathématiciens : on parle désormais d'« analyse numérique ». Il se préoccupe d'obtenir des solutions non par des méthodes "analytiques" (donnant un résultat formel que l'on convertit ensuite en solution numérique en introduisant les paramètres du problème), mais par des méthodes d'approximations successives. Les spécialistes $\mathrm{du}$ calcul numérique cherchent des algorithmes susceptibles de résoudre des problèmes pour lesquels les méthodes formelles sont inefficaces (équations aux dérivées partielles par exemple) ou exigent un temps de calcul rédhibitoire (calcul matriciel). Avant l'arrivée des ordinateurs, ils travaillent à l'aide de calculatrices de bureau,

5 Notamment Raymond, F.-H. L'Automatique des Informations, Masson Paris 1957. Naslin, P. Principes des calculatrices numériques automatiques, Dunod, Notamment Raymond, F.-H. L'Automatique des Informations, Masson Paris 1957. Naslin, P. Principes des calculatrices numériques automatiques, Dunod,
Paris 1958. Sestier, A. Les calculateurs numériques automatiques et leurs applications, Dunod, Paris 1958. Kuntzmann, J. Méthodes numériques - Interpolation - Dérivées, Dunod, Paris 1959. Demarne, P., M. Rouquerol. Les ordinateurs, Puf, Paris 1959. Durand, E., Solutions numériques des équations algébriques, tome 1, Masson, Paris 1960. Boucher, H. Organisation et fonctionnement des machines arithmétiques, Masson, Paris 1960. Pelegrin, M., J.-C. Gille, P. Decaulne. Méthodes modernes d'études des systèmes asservis, Dunod, Paris 1960. 
parfois avec de plus gros matériels, machines à cartes perforées ou simulateurs analogiques : les mathématiciens apprennent à gérer de "grands équipements".

En s'ajoutant à ces machines à partir de 1956, l'ordinateur renouvelle considérablement le domaine, d'abord en offrant des possibilités de calcul infiniment supérieures, mais surtout en suscitant de nouveaux problèmes (méthodes de programmation, architecture des machines, reconnaissance des formes) ; liés à sa spécificité - le programme enregistré, dématérialisé - , ces travaux constituent la base d'une nouvelle discipline, l'informatique. Lorsque des équipes de "numériciens" existent dans une université dans la période 1955-1965, l'acquisition d'ordinateurs est immédiate et débouche sur l'émergence rapide de recherches en informatique. L'existence de recherches en calcul numérique est donc une condition suffisante pour le développement de l'informatique en tant que domaine de recherche dans une université.

C'est aussi quasiment une condition nécessaire. On ne voit guère d'équipe d'informatique se constituer à partir de recherches en électronique, donc de l'aspect matériel des ordinateurs. Et pour cause : aucun projet d'ordinateur n'a vu le jour dans une université française à l'époque pionnière, contrairement à ce qui s'est passé dans les autres pays industrialisés. Cela donne une physionomie particulière, nationale, aux contenus de l'enseignement ; l'informatique universitaire française est centrée sur la programmation et ses aspects mathématiques, l'architecture de machines et la technologie restant notablement négligées ${ }^{6}$. Plus tard, au milieu des années soixante, l'équipe grenobloise de R. Perret se lance bien dans le hardware, mais elle se définit comme une équipe d'automaticiens (aujourd'hui Laboratoire d'Automatique de Grenoble) et c'est dans cette perspective qu'elle développe à partir de 1961 une famille d'ordinateurs industrialisés par Télémécanique. Par ailleurs, le simple achat d'un ordinateur ne débouche pas nécessairement sur un développement de l'informatique : dans bien des cas cette acquisition répond à la demande de disciplines ayant besoin de calcul mais ne prenant pas les méthodes de calcul pour objet. C'est le cas de la mécanique, de l'astronomie, de la physique nucléaire ou de la cristallographie. Enfin, la présence d'enseignements de calcul numérique, ou même d'informatique, n'est un gage de développement de la recherche en informatique que si ces enseignements sont associés à la présence d'un enseignant de rang suffisant dans le système de l'époque, un professeur ou un maître de conférences (équivalent des actuels professeurs de seconde classe), pour organiser une option de troisième cycle et un laboratoire. Les enseignements pratiques, techniques, du calcul sur machines ne suffisent pas.

Cette logique de développement s'illustre bien à partir des cas étudiés pour lesquels on peut à présent donner la trame du développement de l'informatique sous les trois aspects qu'elle peut prendre : outil de calcul, enseignement, recherche.

\section{1 - La recherche en calcul numérique existe avant l'acquisition des ordinateurs : Grenoble, Toulouse, Nancy}

Un cours d'analyse appliquée démarre à l'Institut Polytechnique de Grenoble (IPG) ${ }^{7}$ en 1948, sous la direction d'un jeune professeur, Jean Kuntzmann. Ce cours devient un certificat de licence en 1949. Les travaux pratiques sont faits sur des machines de bureau numériques ou analogiques. En 1952 la faculté achète un calculateur analogique de la Société d'électronique et d'automatisme, qui centuple sa puissance de traitement. Le laboratoire de calcul ne sert pas qu'à l'enseignement, il travaille aussi sous contrat pour des clients extérieurs, nationaux (EDF, CNET, Aéronautique) puis locaux (industrie hydroélectrique). J. Kuntzmann recrute des collaborateurs (6 enseignants et chercheurs en 1958, 53 en 1963) parmi les étudiants de l'IPG. Le laboratoire, promu Institut de Mathématiques Appliquées de Grenoble (IMAG), deviendra l'un des premiers laboratoires associés au CNRS. Il englobe un troisième cycle de mathématiques appliquées en 1956, ainsi qu'une section de mathématiques appliquées pour les élèves-ingénieurs de l'IPG. Les relations que l'IPG entretient avec l'industrie permettent à J. Kuntzmann et à son principal collaborateur, Louis Bolliet, de se former au maniement des premiers ordinateurs installés en 1957 dans des entreprises de la région ${ }^{8}$. Sur cette base, Kuntzmann et Bolliet obtiennent des crédits du Ministère de l’Education nationale (45 MF) pour acheter un Bull Gamma ET en 1957.

À Toulouse, un professeur de physique qui s'intéresse entre autres au calcul numérique, Emile Durand, assure à partir de 1949 des enseignements de calcul qui deviendront un certificat de licence en 1956. Comme à Grenoble, au début, les travaux pratiques sont effectués sur des machines électromécaniques, bientôt sur un calculateur

\footnotetext{
6 Sauf peut-être à toulouse où, bien que l'informatique se soit développée sur la base du calcul numérique, une partie des recherches des années soixante ait été orientée vers la construction d'un ordinateur, la "Calculatrice Automatique de Toulouse".

7 L'Institut polytechnique de Grenoble est alors une fédération d'écoles d'ingénieurs (électronique, électrotechnique, hydraulique, papéterie) partageant les locaux de la faculté des sciences. L'enseignement de Kuntzmann répond d'abord à la demande des électroniciens.

8 “Nous avons commencé à travailler avec une petite entreprise qui avait du matériel Bull. [...] Tout de suite après nous sommes allés à Lyon, à la Compagnie Electromécanique, [...] et puis [...] à Grenoble il y avait Neyrpic [...] qui a acheté un IBM 650. On a travaillé dessus [...] j'ai écrit pas mal de programmes pour M. Kuntzmann qui s'intéressait déjà aux équations différentielles." (L. Bolliet).
} 
analogique de la SEA installé à l'Institut d'Optique électronique (l'informatique se développe toujours sur une base d'équipements préexistants). En 1957, les Toulousains s'adressent directement à IBM qui leur propose un accord très avantageux pour l'installation d'un ordinateur IBM $650^{9}$. Le fait qu'E. Durand ait accédé quatre ans plus tôt à la fonction de doyen a peut-être facilité l'opération. Durand préside par ailleurs le Fonds de recherche du centre de calcul européen d'IBM.

À Nancy en 1958, Jean Legras, professeur de mathématiques enseignant le calcul numérique dans les écoles d'ingénieurs de Nancy, obtient d'IBM l'acquisition d'une calculatrice 604 (programmable par tableau de connexions) pour faire des essais et initier quelques étudiants de troisième cycle. Il démarche l'année suivante les autorités universitaires locales et nationales pour obtenir la location d'un IBM 650 par la faculté des sciences, ce qui ne va pas sans susciter des réticences auprès des mathématiciens "purs". Le Service de Mathématiques Appliquées se renforce par le recrutement de deux enseignants du lycée Poincaré de Nancy ${ }^{10}$.

À Grenoble et Toulouse, les universitaires spécialistes du calcul numérique et des calculateurs n'ont aucun mal à convaincre leurs collègues responsables des écoles d'ingénieurs de créer des filières de "mathématiques appliquées" (le mot "informatique" sera inventé en 1962 pour baptiser une filiale de la SEMA). C'est ainsi que s'ouvrent en 1958/1959 les premières formations spécialisées d'ingénieurs en informatique qui resteront très longtemps les seules du pays (l'Institut d'Informatique d'Entreprise, qui dépend du CNAM parisien, sera créé en 1968, la filière informatique de l'INSA de Rennes en 1967, les écoles de Bordeaux et Lannion en 1986 dans le cadre d'un plan de rattrapage du retard français en la matière). Les enseignements de ces filières, comme c'était courant dans ces écoles, sont en grande partie effectués à l'université dans le cadre des certificats, les travaux pratiques utilisant le centre de calcul qui s'est constitué autour de l'ordinateur ${ }^{11}$.

À partir de la fin des années soixante, l'informatique se structure, finit après bien des difficultés par être reconnue comme discipline à part entière (Mounier-Kuhn, 1987). Dans les trois villes, les chétives équipes initiales se sont fortement étoffées. Bornée au 3e cycle jusqu'en 1966, l'informatique s'intègre à la maîtrise de mathématiques appliquées et fait l'objet de diplômes de 2e cycle grâce à la réforme Fouchet. Des maîtrises d'informatique sont instituées vers 1967 à Toulouse et à Nancy. Des enseignements de niveaux équivalents sont mis en place parallèlement dans les écoles d'ingénieurs et les IUT. Le nombre d'étudiants, d'enseignants et d'ingénieurs croît très rapidement (l'effectif de l'IMAG de Grenoble atteint 99 personnes en 1967).

\section{2 - La recherche en calcul numérique se développe en même temps que l’informatique : Paris et Lille}

Paris est, historiquement, le théâtre des premières recherches sur le calcul en France. De la fin des années trente au milieu des années cinquante, divers organismes scientifiques y ont entrepris de réaliser des machines à calculer. Toutes ces tentatives échouent, pour des raisons variées (Mounier-Kuhn, 1994). La principale, pour le milieu "académique", est celle du CNRS : Louis Couffignal, directeur du laboratoire de calcul numérique de l'Institut Blaise Pascal, conçoit un calculateur électronique, dont la réalisation est confiée à la société Logabax. Couffignal cherche à se démarquer des conceptions de Von Neumann, et s'enferme dans un démarche qui refuse le concept de programme enregistré et la logique séquentielle, préfigurant d'une certaine façon les architectures "parallèles" actuelles (Ramunni, 1988). Il est contraint de quitter ses fonctions en 1957.

Au moment où l'informatique "décolle" à Grenoble et à Toulouse, Paris semble être hors de la course, comme une Formule 1 partie trop vite et qui coule une bielle au troisième tour de circuit. On pourrait imaginer que les ingénieurs et les universitaires ayant échoué à construire du hardware se rabattent sur l'analyse numérique et la recherche en software. En général, il n'en est rien. Les équipes se dispersent, les uns partant dans l'industrie, les

\footnotetext{
9 En fait la machine est donnée, seuls les frais d'entretiens sont à la charge de la faculté : “Si nous avons eu l'IBM 650, c'est grâce aux facilités que nous a accordé IBM. Nous n'avions pas tellement de crédits, et pour eux c'était intéressant. C'était une machine sur laquelle on allait former les gens et, à l'époque, il n'y avait pas beaucoup de machines." (E. Durand)

10 H. Depaix (chef de travaux, 1960) et C. Pair (attaché de recherche CNRS, 1964). H. Depaix se spécialise dans l'enseignement des statistiques et C. Pair dans la programmation (il avait assisté dès 1958 à un séminaire de J. Kuntzmann à l'Institut d'Astrophysique, dans le cadre de l'association Française de Calcul (Hoffsaës, 1988). C. Pair sera le principal animateur de la recherche en informatique à Nancy. Par ailleurs, il faut signaler qu'à cette époque existe un autre ordinateur à l'université, le Gamma 60 du "Trésor de la Langue Française" (entreprise du CNRS pour réaliser un dictionnaire informatisé), mais les mathématiciens n'y ont quasiment pas accès : “c'était totalement disjoint. Je sais qu'à l'époque, Legras aurait bien voulu travailler sur le Gamma 60, malheureusement, ça ne s'est pas fait" (H. Depaix).

11 Les ordinateurs étant une ressource rare, il ne peuvent être réservés aux seuls spécialistes du calcul numérique. Le ministère et les autorités universitaires locales imposent donc la constitution de centres de calculs au service des communautés scientifiques locales et des éventuels utilisateurs extérieurs (services administratifs, industriels). Dans cette période, la distinction entre l'outil et le domaine de recherche est faible. Les laboratoires de calcul qui se mettent en place ont au début dans leurs attributions la gestion des centres. Ce sont les mêmes personnes qui font de la recherche, assurent les cours d'analyse numérique ou de programmation, gèrent le centre de calcul et les relations avec les différents utilisateurs. Cette unité éclatera au cours des années soixante-dix (dès 1969 au CNRS) les premiers centres d'informatique étant devenus de très grands ensembles (parfois plus de cent personnes), les autorités administratives pousseront à leur éclatement en unités plus facilement gérables ; à la même époque, l'informatique sera reconnue comme un domaine de recherche autonome et majeur, ce qui justifiera de séparer ses chercheurs et ses unités de service. Vingt ans plus tard, l'existence de celles-ci est mise en question par la large diffusion des ordinateurs personnels.
} 
autres changeant totalement de discipline. Couffignal se consacre à une cybernétique de plus en plus fumeuse. L'Institut Henri-Poincaré (faculté des sciences) aurait vocation à devenir le berceau de l'informatique parisienne, si le principe n'avait été posé en 1942 qu'il laisse au CNRS le monopole des équipements lourds de calcul. Seuls subsistent les bureaux de calcul de l'Institut Henri-Poincaré et de l'Institut Blaise-Pascal, exécutant des travaux pour la communauté scientifique. Un groupe de calcul numérique s'est bien formé à l'Institut Henri-Poincaré dès 1947, mais s'il organise des séminaires rassemblant différents spécialistes, il ne constitue ni un laboratoire ni un centre d'enseignement. L'Institut Blaise Pascal achète en 1955 un ordinateur Elliott 402 importé de Grande Bretagne, mais aucune activité d'enseignement ou de recherche ne se développe autour de cette machine ('IBP est un laboratoire propre du CNRS, installé hors de tout environnement universitaire).

Ce sont donc des hommes nouveaux qui, dans la seconde moitié des années cinquante, relancent Paris dans le jeu - au moment où l'industrie produit les premiers calculateurs commerciaux de série. En 1956, le Conseil supérieur de la recherche scientifique et du progrès technique ${ }^{12}$, dont un comité rassemble les spécialistes française du calcul et de la "Cybernétique", incite le Ministère de l'Education nationale à instaurer trois certificats de calcul numérique dans trois facultés des sciences. Les deux premiers officialisent les enseignements déjà assurés à Grenoble et à Toulouse par Kuntzmann et Durand. Le troisième est confié à un professeur de la Sorbonne, Jean Ville. Parallèlement, le directeur du CNRS fait venir d'Alger son ancien camarade de taupe, René de Possel, un brillant mathématicien reconverti du bourbakisme aux mathématiques appliquées. Il l'impose d'abord à la tête du laboratoire de calcul de l'Institut Henri-Poincaré, puis comme successeur de Couffignal à l'Institut Blaise Pascal. Enfin, dans des circonstances encore mal connues, c'est pour de Possel, non pour Jean Ville, qu'une chaire d'analyse numérique est créée en 1959 à Paris. Complétant ce dispositif (calcul, recherche, enseignement universitaire), de Possel fonde en 1962 un institut de programmation destiné à former des ingénieurs en informatique. L’Institut Blaise Pascal sera dissous en 1969 (Mounier-Kuhn, 1988, 1989).

À Lille, les débuts de l'informatique découlent d'une réorganisation complète de l'enseignement des mathématiques, amorcée par l'arrivée de jeunes professeurs issus de l'Ecole normale supérieure. L'un d'eux (Georges Poitou) décide en 1959 de mettre en place un cours de calcul numérique, assuré par un intervenant extérieur, ingénieur chez IBM. Pour compléter cet enseignement par des TP, il est nécessaire de s'équiper en machines. L'un des nouveaux responsables du département de mathématiques (Georges Parreau), élu doyen en 1961, n'a aucune difficulté pour mobiliser des crédits - le rapport de conjoncture du CNRS, suivi par la DGRST, a recommandé d'équiper plusieurs universités en ordinateurs, et le IVe Plan a prévu les crédits nécessaires. La faculté acquiert d'abord des machines électromécaniques puis un Bull Gamma ET, autour duquel s'organise un laboratoire de calcul, dirigé par G. Poitou. La faculté bénéficie à ce moment-là de l'arrivée de deux enseignants de Strasbourg, dont l'un, astronome formé à l'utilisation du Gamma ET (P. Bacchus), s'occupera du centre de calcul, et l'autre (P. Pouzet), mathématicien, prendra en charge les enseignements de ce domaine ainsi que l'essentiel de la recherche en informatique. Une licence d'informatique sera instituée en 1968, le troisième cycle s'organisant progressivement autour des laboratoires.

\section{2 - Logiques exogènes : rattraper le retard}

Les pôles précurseurs étant constitués, ils bénéficient à la fin des années soixante d'un avantage important. Leurs responsables contrôlent le développement de la discipline au sein des instances nationales : ils siègent à la DGRST et au Commissariat général du Plan (J. Kuntzmann préside le comité d'action concertée "Calculateurs" et le groupe "Applications aux machines à calcul" de la Commission permanente de l'électronique du Plan), au CCU, à la DRME, au Comité national de la recherche scientifique ${ }^{13}$ (Mounier-Kuhn 1987) ; l'Institut de Recherche en Informatique et Automatique, créé en 1966 dans le cadre du Plan Calcul, est dirigé par M. Laudet. Ils disposent des plus puissants ordinateurs, facteur attractif important vis-à-vis des chercheurs. En croissance continue, ils absorbent eux-mêmes les docteurs qu'ils ont formés, rendant très difficile l'essor de nouveaux pôles. Un rééquilibrage s'amorce à partir de 1970 lorsqu'ils ne peuvent plus intégrer tous leurs jeunes chercheurs : leur croissance atteint nécessairement un palier, comme l'ensemble de la recherche et de l'enseignement supérieur à l'époque ; le Plan Calcul favorise la multiplication des centres d'informatique, permettant à diverses universités d'offrir des situations attrayantes. Des essaimages commencent alors, essentiellement à partir de Grenoble et de Paris, à la demande d'universités désireuses de combler leur retard en la matière ou à l'initiative des "essaimés"

\footnotetext{
12 Le CSRSPT (Conseil supérieur de la recherche scientifique et du progrès technique), instance consultative créée sous le gouvernement Mendès-France et présidée par Henri Longchambon, est remplacé en 1959 par la DGRST (Délégation générale à la recherche scientifique et technique) qui dispose de pouvoirs beaucoup plus étendus. Voir A. Prost "Les origines de la politique de recherche en France (1939-1958)" Cahiers pour l'Histoire du CNRS n ${ }^{\circ}$, Editions du CNRS.

13 Entre 1963 et 1970, pendant deux mandats du Comité national, l'informatique est représentée par sept parisiens, quatre grenoblois, trois toulousains, un nancéen et deux "autres" (un bisontin, un rennais).
} 
eux-mêmes. L'analyse de différents cas permet de saisir d'une part les logiques qui y ont entravé le développement de la recherche en informatique dans la phase initiale, d'autre part les processus de rattrapage ultérieurs.

\section{1 - Des débuts avortés : Strasbourg et Rennes-Nantes}

À Strasbourg et dans le bipôle Rennes-Nantes, l'informatique semble se développer d'abord comme dans les pôles précurseurs, mais une rupture à un moment clé du processus fait avorter celui-ci. Rennes prend un bon départ, mais c'est finalement selon une logique exogène, après une période transitoire, que l'informatique $\mathrm{y}$ prendra un véritable essor. À Strasbourg, l'arrêt du développement endogène provoque une stagnation durable de la recherche en informatique.

Le directeur de l'Observatoire Astronomique de Strasbourg, P. Lacroute, entrevoit à la fin des années cinquante la possibilité d'utiliser les ordinateurs qui arrivent alors sur le marché, pour effectuer les calculs très complexes exigés en astrométrie. Il prend contact avec Bull et obtient la location à un prix modéré d'un calculateur Gamma 3. Pour faire fonctionner la machine, P. Lacroute recrute des collaborateurs techniques ainsi qu'un jeune astronome, P. Bacchus, qui se passionne pour la programmation et rejoint la petite équipe ${ }^{14}$. S'y ajoute P. Pouzet, un mathématicien spécialiste du calcul numérique (il achève une thèse sous la direction de J. Kuntzmann à Grenoble), sollicité par P. Lacroute pour coordonner les travaux réalisés sur la machine et instaurer un certificat de calcul numérique. Le "Centre de Calcul Électronique" de la Faculté des Sciences est inauguré le 22 Novembre 1960 .

Ainsi, entre 1959 et 1961, les structures mises en place à Strasbourg sont très semblables à celles qui existent depuis peu à Grenoble et à Toulouse : un ordinateur géré par une équipe technique au service de la communauté scientifique ; des enseignements de mathématiques appliquées ; des travaux de recherche avec la thèse de $P$. Pouzet. Mais une importante bifurcation se produit quand partent P. Bacchus en 1961, P. Pouzet en 1962. Bacchus obtient une chaire à Lille ; Pouzet, qui n'a pu, semble-t-il, obtenir un poste de professeur à Strasbourg après la soutenance de sa thèse ${ }^{15}$, décide de rejoindre son collègue à Lille où sa venue est sollicitée par les mathématiciens locaux, qui viennent de créer des enseignements de mathématiques appliquées et d'acquérir un ordinateur (voir plus haut)

Le départ simultané de ces deux chercheurs pose un certain nombre de problèmes. Dans le certificat de calcul numérique, on doit les remplacer par divers enseignants venus d'autres universités ou d'autres disciplines. Ce départ sonne pour un temps le glas de la recherche qui aurait nécessité la création d'un poste de professeur de calcul numérique. Une tentative de rattrapage a lieu en 1967. Les responsables du département de mathématiques demandent la création d'un poste de maître de conférences en informatique, mais n'obtiennent qu'une habilitation à la maîtrise ${ }^{16}$ qu'ils doivent organiser avec les moyens du bord. Le troisième cycle démarrera seulement dans les années quatre-vingt avec l'arrivée d'un ancien de l'IMAG, J.-C. Lafon ; des enseignants locaux fonderont en 1985 le Centre de Recherches en Informatique, reconnu comme jeune équipe CNRS en 1990.

En 1955, Nantes n'a pas d'université mais une école d'ingénieurs, l'Ecole Nationale Supérieure de Mécanique (ENSM), dépendant de la Faculté des Sciences de Rennes. L'un des deux professeurs de mathématiques de l'ENSM, Georges Brillouët, s'intéresse au calcul numérique en partie pour répondre aux besoins de la formation des ingénieurs. G. Brillouët est nommé professeur d'analyse numérique à la faculté de Rennes en 1959 et obtient la création d'un certificat d'analyse numérique en 1960. Parallèlement, l'ENSM cherche à s'équiper en moyens de calculs et reçoit en 1960 des crédits pour l'acquisition d'un IBM 650.

Paradoxalement, c'est l'ouverture en 1961 d'une Faculté des sciences à Nantes qui porte un coup d'arrêt au développement de l'informatique locale, car G. Brillouët en est nommé doyen : "Mes fonctions de Doyen d'une Faculté nouvelle ont alors pris le pas sur mes activités informatiques. Il fallait assurer les enseignements

\footnotetext{
14 “J'avais été immédiatement séduit par ce nouvel engin, et je me suis rapidement mis à y passer mes journées, voire mes nuits, tant pour les travaux astronomiques que pour collaborer avec les uns et les autres - et aussi pour apprendre cette nouvelle discipline. Je n'avais évidemment d'autre formation que ma culture générale scientifique ( $\mathrm{P}$. Bacchus est normalien, agrégé de physique), et j'ai tout appris sur le tas. Pouzet, qui avait suivi je crois un stage de formation à l'ordinateur, m'y a aidé ; les techniciens de Bull, qui étaient en permanence derrière la machine, m'ont été très précieux" (P. Bacchus).

15 P. Pouzet n'a pu être interrogé, mais il semble que les responsables de la Faculté n'étaient pas favorables au recrutement d'un spécialiste des mathématiques appliquées.

16 Il semble que la demande a été mal formulée ou mal comprise : "Le département de mathématiques a décidé que [...] ce serait bien d'avoir une maîtrise de conférence d'informatique [...] L'ennui c'est qu'ils ont demandé une maîtrise d'informatique [...] la demande est partie avec "maitrise d'informatique" et nous est revenue" (R. Strosser, enseignant d'informatique). Les maîtrises d'informatique venaient d'être créées par la réforme Fouchet, en même temps que les maitrise d'électronique et que les IUT.
} 
fondamentaux, et, ce qui compliquait les choses, il y avait à l'époque une sérieuse crise du recrutement: listes d'aptitudes presque vides, peu d'enthousiasme à venir jouer les pionniers, etc. " (G. Brillouët).

À la suite de ce changement, c'est plutôt à Rennes que l'informatique se développe, nettement plus tard, sur la base des cours de calcul numérique. L'un des enseignants de mathématiques appliquées, Jean Céa, inaugure en 1969 un enseignement d'informatique avec l'aide de jeunes assistants et d'intervenants grenoblois. En 1970 s'ouvre une maîtrise d'informatique ; la petite équipe rennaise bénéficie de l'arrivée de 8 informaticiens dont 6 grenoblois sur des postes d'enseignement ou d'ingénieurs du centre de calcul. À partir de là, l'informatique connaît un essor rapide à Rennes, qui bénéficie par ailleurs de la décentralisation en Bretagne d'activités du CNET, puis de l'IRIA. L'actuel Institut de recherche en informatique et systèmes aléatoires (IRISA) rassemble actuellement $11 \%$ des chercheurs ou enseignants-chercheurs de la discipline intégrés dans une unité CNRS.

Nantes se spécialise plutôt en Automatique (renouvellement assez naturel de l'école de mécanique), autour de R. Menzecev dont l'équipe "Identification et optimalisation par voie hybride" est associée au CNRS en 1968.

\section{2 - Des ordinateurs ou des enseignements sans équipe de recherche : Bordeaux et Montpellier}

Dans ces deux villes, des utilisateurs de moyens de calcul non mathématiciens - astronomes, cristallographes, physiciens nucléaires - sont à l'origine des premières acquisition de machines. Mais l'absence d'enseignant titulaire (professeur ou maître de conférence) en calcul numérique a eu pour effet un développement tardif de la recherche et parfois des enseignements en informatique.

À Bordeaux, les cristallographes ont de gros besoins de calcul. Ils s'associent avec les mathématiciens en 1961 pour acquérir un IBM 1620, qui est installé en physique. Parmi les mathématiciens, seul un jeune assistant, J. Hardouin Duparc, s'y intéresse et commence à programmer. D'autres disciplines en devenant clientes, un service de calcul de la faculté est officiellement créé en 1967. Des cours de programmation sont organisés pour les utilisateurs. Ils sont assurés par deux ingénieurs militaires du Centre d'essais des Landes, non par des universitaires. Le service de calcul accédera en 1973 au statut de centre interuniversitaire. L'enseignement du calcul numérique se développe peu malgré la présence d'un spécialiste, J.-M. Blondel, affecté essentiellement à des enseignements de premier cycle et à la préparation aux concours de recrutement des enseignants. Un certificat de calcul numérique, créé en 1963, est assuré par un intervenant extérieur et ne comporte pas de travaux pratiques ${ }^{17}$.

Les autorités politiques locales prennent conscience à la fin des années soixante du retard de leur université en informatique et effectuent diverses démarches qui aboutissent à la création d'une chaire dans cette discipline en 1970. Les mathématiciens locaux se tournent vers les équipes reconnues, celle de Kuntzmann à Grenoble et celle qu'animent M.-P. Schutzenberger et J.-L. Lions à Paris. Finalemnt, c'est un élève de ce dernier, Y. Haugazeau, alors maître de conférences à Lyon, qui est nommé sur ce poste. À son arrivée il partage avec J.-M. Blondel les enseignements de mathématiques appliquées, crée la licence d'informatique, et participe à la mise en place du groupe d'analyse appliquée et d'informatique. Sa première préoccupation est de recruter de "vrais" spécialistes de l'informatique, mais cela ne se révèle guère aisé : "Dans les trois centres principaux, Paris, Grenoble et Toulouse, les gens n'avaient guère envie de partir. Quand j'ai voulu recruter, j'ai joué les voyageurs de commerce" (Y. Haugazeau). Finalement deux grenoblois, J.-L. Joly et A. Bouchet, ainsi qu'un enseignant de l'INSA de Lyon, P. Morel, sont recrutés comme maîtres de conférences, J. Hardouin Duparc étant mis à contribution pour les travaux pratiques. Une équipe d'informaticiens se constitue pendant que les mathématiques appliquées se renforcent. Désormais, l'informatique se développe rapidement : mise en place d'une MIAGE en 1982, d'une formation d'ingénieurs ${ }^{18}$, d'un laboratoire et d'un DEA en $1986^{19}$.

À Montpellier, c'est aussi des cristallographes que vient l'initiative d'acquérir un ordinateur. Ne pouvant accéder facilement aux calculateurs de Grenoble et Toulouse, souvent saturés, l'un d'entre eux, J. Falgueirettes, se bat pour obtenir la création d'un centre de calcul à Montpellier. Le changement d'équipe dirigeante de la Faculté des Sciences lui permet d'obtenir les appuis nécessaires et un IBM 1620 est installé en 1961.

\footnotetext{
17 Ce faible développement du calcul numérique malgré la présence d'un spécialiste s'explique en partie par le fait que le responsable du département de mathématiques (J. Colmez) n'était guère favorable aux mathématiques appliquées.
}

18 Bordeaux bénéficie alors d'un plan de rattrappage du retard français dans la formation des informaticien, qui aboutit en 1986 à créer deux nouvelles filières d'informatique, au sein des écoles d'ingénieurs existantes de Bordeaux et Lannion. Signalons par ailleurs qu'un IUT d'informatique a été créé en 1970 à l'initiative d'enseignants de gestion, mais n'a eu longtemps que peu de rapport avec les informaticiens de la faculté des sciences, si ce n'est par la participation des enseignants de l'IUT aux séminaires.

19 Les informaticiens ont longtemps fait partie du Laboratoire de Mathématiques et d'Informatique avant de prendre leur autonomie en 1986 pour créer un DEA autonome et former le Laboratoire Bordelais de Recherche en Informatique (LABRI), reconnu par le CNRS en 1988. Le nombre des informaticiens a augmenté doucement mais régulièrement jusqu'en 1981/1982 avant de connaître une croissance soutenue pour atteindre en 1988 un total de 46 titulaires. 
La création d'un IUT d'informatique en 1967 marque le début d'un enseignement dans ce domaine et favorise le contact avec le secteur privé, notamment avec IBM qui dispose d'un important établissement à Montpellier. C'est sur les conseils des ingénieurs d'IBM que les enseignants élaborent un projet de formation à l'informatique de gestion qui sera accepté par le Ministère en 1968 et deviendra l'Institut des Sciences de l'Ingénieur de Montpellier (ISIM). Ce développement important au niveau des enseignements s'effectue en recourant à des intervenants extérieurs ou à des universitaires de disciplines proches de l'informatique (statisticiens par exemple). Mais il n'a aucune retombée sur la recherche, inexistante jusqu'à la fin des années soixante-dix. Le calcul numérique ne se développe pas malgré les tentatives de J. Falgueirettes qui obtient dès 1962 des heures complémentaires pour assurer des enseignements dans ce domaine, mais ne trouve pas de candidats pour venir occuper un poste de maître de conférence ou de professeur. Les jeunes docteurs de Grenoble et de Toulouse sont très réticents à s'installer dans un contexte où tout est à créer et où les moyens de calcul sont relativement faibles. Il faudra attendre le début des années quatre-vingt pour voir arriver de Paris VI une partie du Groupe de Recherche 22, qui décide unilatéralement de chercher une implantation en province ${ }^{20}$. Les parisiens ainsi transplantés, auxquels se joignent quelques enseignants de l'IUT, forment le noyau de la première véritable équipe de recherche en informatique, le Centre de Recherche en Informatique de Montpellier (CRIM), qui s'est associé à une équipe de robotique pour former en 1992 le LIRM (Laboratoire d'informatique et de robotique de Montpellier). Cette même équipe est à l'origine de la création de la filière d'enseignement d'informatique (licence, maîtrise, DEA) entre 1983 et 1985.

\section{3 - La découverte tardive de l'informatique : Lyon et Marseille}

Certaines facultés, n'ayant pas acquis d'ordinateurs au début des années soixante, tentent de rattraper leur retard, de leur propre initiative ou à la suite d'incitations du ministère, après que l'informatique ait conquis une légitimité. Les responsables de la faculté invitent des spécialistes parisiens ou grenoblois à y mettre en place à la fois des enseignements, de la recherche et un centre de calcul.

Le démarrage tardif de l'informatique à Lyon s'explique en grande partie par la proximité du pôle grenoblois. Le premier contact de la Faculté des Sciences de Lyon avec l'informatique s'opère en 1965 lorsque le responsable du département de mathématiques, J. Braconnier, fait venir un membre de l'Institut Blaise Pascal, Claude-F. Picard. Celui-ci met en place un certificat d'informatique appliquée et commence à constituer une petite équipe de recherche autour d'un IBM 1620. Père de six enfants, C.-F. Picard ne parvient pas à faire suivre sa famille, et repart à Paris en 1967 avec la plupart des membres de son équipe. Tout est à recommencer. Un autre parisien, Yves Haugazeau, lui succède mais quitte Lyon en 1969 pour occuper une chaire à Bordeaux (voir plus haut). L'arrivée en 1970 d'un grenoblois, M. Terrenoire, permet enfin à l'informatique de se développer : organisation d'un centre de calcul et d'une maîtrise d'informatique appliquée à la gestion en 1972, puis mise en place progressive d'une filière complète au cours des années quatre-vingt.

Marseille a abrité, d'une certaine façon, le premier laboratoire français de calcul, puisque, dès les années 1930, Joseph Pérès et Lucien Malavard y avaient réalisé des calculateurs analogiques pour l'aérodynamique, dans le cadre du Centre de recherches scientifiques, industrielles et maritimes. Pourtant, c'est seulement au milieu des années soixante que les mathématiciens marseillais créent un certificat de calcul numérique, confié à un ancien élève parisien de J.-L. Lions, H. Morel, recruté en 1962 comme enseignant. La faculté reçoit des crédits pour fonder en 1966 un centre de calcul autour d'un calculateur SETI Pallas, sur lequel les mathématiciens locaux feront des travaux pour la Marine nationale. En 1968 est créée la faculté de Luminy où se met en place un département de mathématiques et d'informatique. Le ministère incite le responsable de ce département à recruter un informaticien. A. Colmerauer, ingénieur ENSIMAG (Grenoble), alors en poste à Montréal, est nommé maître de conférence en 1971 et forme une petite équipe marseillaise avec de jeunes chercheurs locaux. Cette équipe se distinguera par la suite en concevant le langage PROLOG, mondialement utilisé en intelligence artificielle. Un peu plus tard, d'autres grenoblois partiront former une équipe à Nice.

\section{3 - Ecoles d'ingénieurs et calcul numérique}

L'analyse des différents cas montre qu'un développement endogène et précoce de la recherche en informatique est toujours lié à la présence d'équipes de recherche en calcul numérique. Le problème de la différenciation

20 “Nous nous sommes dit “Où avons-nous envie d'aller ?”. [...] On hésitait entre Marseille et Montpellier” (M. Chein, membre du GR22) 
géographique est alors renvoyé en amont : pourquoi trouve-t-on de telles équipes à Grenoble, Toulouse, Nancy, Lille et Paris et non ailleurs ${ }^{21}$ ?

Ce qui a été décrit pour les facultés où la recherche en informatique s'est développée de mannière exogène montre l'existence de logiques sociales qui s'opposent au développement de la nouvelle discipline qui, en dehors du calcul numérique, ne trouve pas de cadre institutionnel et continue à être considérée comme un simple instrument (Bordeaux, Montpellier, Strasbourg). Pour saisir pleinement les logiques qui expliquent les différences entre les cas étudiés, il est nécessaire de revenir sur les cinq pôles précurseurs en distinguant deux cas de figure :

- soit le calcul numérique et l'informatique sont développés de façon volontariste dans le cadre d'une réorganisation des mathématiques (Lille) ou à cause de "l'effet capitale" (Paris),

- soit la présence d'écoles d'ingénieurs dans les facultés des sciences impose le recrutement de spécialistes du calcul numérique (cas de Grenoble, Toulouse et Nancy). Dans le premier cas, on se trouve face à une situation spécifique, en grande partie contingente (notamment à Lille), alors que dans le second c'est la configuration institutionnelle du système scientifique local qui explique le développement des disciplines appliquées.

\section{1 - Une volonté de développer les mathématiques appliquées : Paris et Lille}

Le cas de Lille est le seul où la création d'enseignements de mathématiques appliquées vient des mathématiciens "purs". Ce phénomène semble s'expliquer par le contexte de complète réorganisation de la Faculté et par l'arrivée de jeunes enseignants issus de l'École normale supérieure ${ }^{22}$. Une fois en place, la jeune équipe de mathématiques décide de constituer des enseignements de mathématiques appliquées, ce qui est présenté par un acteur de l'époque comme un choix scientifique : "Poitou avait toujours eu le goût des mathématiques appliquées. Les mathématiciens de ma génération étaient très conscients que nos disciplines étaient très théoriques et qu'il y avait besoin de développer les mathématiques appliquées" (G. Parreau). Pour le calcul numérique on fait appel à un normalien travaillant pour la firme $\mathrm{IBM}^{23}$.

À Paris, les choses sont un peu différentes. Les premiers enseignements de calcul numérique en Sorbonne sont dispensés par J. Ville, un spécialiste des probabilités et de la théorie de l'information un peu en marge de la communauté mathématique de l'époque, mais d'une grande stature scientifique et qui travaille avec l'industrie (SACM-Alcatel) ; il est conscient de la montée générale des besoins de calcul. Parallèlement, les avatars de l'Institut Blaise Pascal et la nomination à sa tête du mathématicien de Possel aboutissent au choix de celui-ci pour inaugurer une chaire d'analyse numérique parisienne en 1959. Un "effet capitale" joue probablement : la présence d'une extraordinaire concentration de scientifiques et le prestige de la capitale font qu'une nouvelle discipline, si peu valorisée soit-elle, doit y être enseignée. Mais, étant donnée la domination absolue des mathématiques pures sur la scène parisienne de l'époque, cette discipline sera maintenue au rang que lui attribue l'échelle de valeurs bourbakiste : le dernier. De Possel ? Il a été l'un des fondateurs du groupe Bourbaki, mais depuis "il a cessé de faire des mathématiques" ; il sera blackboulé à l'Institut. L'institut de programmation ? Pas question d'accueillir çà sur le territoire universitaire ; il sera abrité par le CNRS dans une ancienne usine de la CSF, jusqu'en 1968 où il est admis sur le nouveau site de Jussieu.

\section{2 - Le poids des écoles d'ingénieurs à Grenoble, Toulouse et Nancy}

À l'époque considérée, les Facultés des sciences de Grenoble, Toulouse et Nancy ont pour point commun d'être dotées d'importantes écoles d'ingénieurs, en particulier dans les domaines de l'électricité (électrotechnique, électronique) et de la mécanique des fluides. Dans ces domaines, les ingénieurs ont de plus en plus besoin de maitriser les techniques des mathématiques appliquées. Ainsi, dans un "vœu" adressé en Avril 1950 aux diverses

\footnotetext{
21 Les débuts avortés de Nantes et Strasbourg montrent l'importance des événements contingents dans un contexte où les acteurs concernés sont en petit nombre et où c'est parfois sur un seul individu que repose la possibilité d'une bifurcation institutionnelle. Le cas de Nantes illustre parfaitement ce fait dans la mesure où la nomination de Brillouët à la fonction de Doyen n'a pas de rapport direct avec le problème qui nous concerne mais provoque une sorte de "catastrophe" dans le développement de l'informatique au sein du bipôle Rennes-Nantes. Le cas de Strasbourg est plus ambigü : il est fort probable que Pouzet n'a pu obtenir immédiatement un poste de maître de conférences entre autres à cause de l'opposition des mathématiciens locaux, ce qui rejoint la logique d'opposition des mathématiciens "purs" aux mathématiques "appliquées" que nous retrouvons plus loin dans le cas de Nancy, mais avec une issue différente.

22

"Nous sommes arrivés, trois copains en même temps. Descombes est arrivé le premier en 1954 comme assistant. C'est un camarade de promotion de G. Poitou. Poitou est arrivé l'année suivante [...] Et comme nous étions très liés (ils avaient tous deux été attachés de recherche au CNRS et représentants des chercheurs à la commission de mathématiques pures), il a souhaité que je vienne ici. [...] Nous arrivions, avec à peu près le même âge et toutes les places étaient devant nous. Les effectifs étaient en pleine croissance : je me souviens d'avoir vu le responsable de la chaire de calcul en 1957-58, complètement effaré à une rentrée, me dire qu'il avait 60 étudiants alors qu'il en avait 12 l'année précédente. Nous avons recruté, fait créer un centre d'enseignement de géométrie supérieure, un enseignement de probabilités, des enseignements d'algèbre ..." (G. Parreau)

23 “Il s'est trouvé que dans la promotion de Poitou, il y avait un normalien qui s'était détourné de l'enseignement, Jean-Claude Hertz. [...] Il est devenu l'un des responsables du secteur scientifique d'IBM [...] Il était très désireux de garder des contacts avec l'enseignement, et comme il n'était pas très loin de Paris, il a joué le rôle de professeur associé. C'est vraiment lui qui a lancé le calcul numérique ici." (R. Parreau).
} 
instances concernées (ministère, écoles d'ingénieurs, sociétés savantes, revues spécialisées), le Président de la Société française des électriciens, M. Ponte, demande à ce que les écoles d'ingénieurs électriciens renforcent leurs programmes de mathématiques appliquées et prévoient, parallèlement à l'enseignement théorique, un enseignement pratique approfondi du calcul numérqiue, graphique et mécanique ${ }^{24}$. Il existe donc une demande sociale pour les enseignements de mathématiques appliquées dans les écoles formant aux métiers de l'électricité.

Les écoles de Grenoble, Toulouse et Nancy, baptisées Écoles nationales supérieures d'ingénieurs (ENSI) en 1948, sont internes aux Facultés : elles n'ont pas de corps professoral autonome et doivent donc recourir à celui de la Faculté 25 . Lorsque leurs responsables, universitaires eux-mêmes, veulent recruter un enseignant, ils doivent obtenir l'accord de leurs collègues de la Faculté. Cette caractéristique a pour effet à Grenoble et Nancy le recrutement précoce d'un professeur de calcul numérique, soit avec l'accord des mathématiciens (cas de Grenoble), soit contre leur avis (cas de Nancy). Toulouse constitue un cas particulier dans la mesure où si les écoles d'ingénieurs sont étroitement associées au développement de l'informatique en fournissant de nombreux étudiants de thèse et en mettant en place une filière de formation du même type que celle de Grenoble, elles ne sont pas forcément à l'origine du recrutement d'E. Durand ${ }^{26}$.

A Grenoble, J. Kuntzmann, qui est un mathématicien "pur” (normalien préparant un doctorat en algèbre), engagé par l'université dès 1942 (il est alors prisonnier de guerre et ne prendra effectivement ses fonctions qu'en 1945) est sollicité dès son arrivée par le responsable des formations d'ingénieurs pour enseigner le calcul numérique : "Revenant à la vie civile après cinq années de guerre et de captivité, il ne me semblait pas évident que je devais reprendre mes activités mathématiques là où je les avais laissées, c'est-à-dire dans une algèbre désincarnée. J'étais donc assez réceptif aux appels extérieurs. Or, le directeur de l'IPG, M. Esclangon, désirait développer dans cette école l'enseignement mathématique." (Kuntzmann, 1992). J. Kuntzmann ne se contente pas d'assurer des enseignements pour les ingénieurs, il oriente résolument ses travaux de recherche vers les mathématiques appliquées et le calcul numérique.

Le problème est réglé à Toulouse par le recrutement d'un physicien compétent dans cette matière, ce qui n'enlève donc pas de poste aux mathématiciens tout en permettant la mise en place d'enseignements de calcul numérique. E. Durand, normalien, élève de L. de Broglie, était réputé pour ses travaux en mécanique ondulatoire et en physique mathématique, discipline proche du calcul numérique. Sollicité par les physiciens locaux, en particulier G. Dupouy, directeur du laboratoire d'Optique électronique ${ }^{27}$, il est nommé sur une chaire de physique théorique en 1949 et commence à professer le calcul.

Les écoles d'ingénieurs de Nancy cherchent aussi à mettre sur pied des enseignements de calcul numérique, mais se heurtent aux mathématiciens locaux parmi lesquels dominent les "Bourbakistes", a priori peu intéressés par les applications. En 1951, les responsables des écoles veulent faire nommer un spécialiste des mathématiques appliquées, Jean Legras, alors que les mathématiciens locaux, appuyés par la majorité des professeurs de la faculté, défendent la candidature de J.-P. Serres, considéré alors comme le meilleur mathématicien de sa génération (il sera nommé au Collège de France à 30 ans). Un bras de fer oppose les responsables des écoles et de la faculté à l'ensemble de la communauté mathématique française de l'époque, principalement composée de normaliens. Finalement, les partisans des mathématiques appliquées ont gain de cause, notamment grâce à l'intervention décisive du directeur de l'Ecole nationale supérieure d'électricité et de mécanique, Georges Goudet : “(Les directeurs d'écoles) ont insisté auprès du ministère [...] et j'ai été nommé contre l'avis des [...] mathématiciens de Nancy. Dès que je suis arrivé, j'ai fait des cours à l'école d'électromécanique, à l'école de brasserie" (J. Legras). J. Legras ouvre aussi des enseignements de licence puis de troisième cycle.

\footnotetext{
24 Archives du CNAM, dossier "Chaires de Mathématiques 1950". Cette demande débouchera aussi sur la création d'un enseignement de mathématiques appliquées au Conservatoire National des Arts et Métiers (P-E. Mounier, Kuhn, "notice biographique d'Alexius Hocquenghem", à paraitre dans les Cahiers d'Histoire du $C N A M, \mathrm{n}^{\circ} 6$ )

25 Les ENSI peuvent seulement, sur leurs ressources propres, rémunérer des intervenants extérieurs et des chargés de cours.

26 Il y a toutefois un lien entre l'école et les mathématiques appliquées. L'ENSEEHT étoffe en effet dès 1945 les enseignements de mathématiques, les cours étant assurés par R. Huron, qui fera par la suite une thèse en mécanique des fluides, se spécialisera en statistiques, fondant le Laboratoire de Probabilité et Statistiques en 1951, et occupera la première chaire de mathématiques appliquées de Toulouse en 1956. C'est ainsi sur l'autre versant des mathématiques appliquées, les statistiques, et non sur le calcul numérique que la présence de l'ENSEEHT a des effets. On peut penser que si E. Durand n'avait pas été là, la Faculté aurait dû recruter un enseignant pour le calcul numérique.
}

27 G. Dupouy est alors le "patron" de la Faculté des Sciences (il sera par la suite directeur du CNRS de 1950 à 1957). Spécialiste d'optique électronique, il a dirigé la construction à Toulouse d'un microscope électronique, centre de son laboratoire. La venue d'un théoricien comme E. Durand devait compléter le département de physique de Toulouse dont le point fort était pluptôt la recherche empirique menée par l'équipe de Dupouy. Durand devait aussi renouveler les enseignements d'électricité et de mécanique quantique. Ses compétences en calcul numérique n'ont donc semble-t-il pas joué un rôle déterminant dans sa venue à Toulouse. 


\section{3 - Logiques de disciplines, logiques d'institutions et systèmes scientifiques locaux}

Pour expliquer ce qui s'est passé dans les différents sites, il est nécessaire de se replacer dans le système universitaire de l'époque avec ses acteurs dominants et ses enjeux spécifiques.

Bien que la vie universitaire implique différentes catégories professionnelles (administratifs, techniciens, assistants), l'essentiel des enjeux est concentré pour la période étudiée sur un type d'acteur détenant tout le pouvoir, les universitaires de haut rang, maîtres de conférences et surtout professeurs. Ils sont les seuls à disposer d'un réel pouvoir d'initiative et de décision permettant d'ouvrir un nouvel enseignement ou de créer une équipe de recherche. La plupart sortent de l'École normale supérieure qui détermine en grande partie leurs références, leurs valeurs ainsi que les relations sociales qui structurent leur milieu professionnel. Au sein de cette communauté relativement homogène, les identités se construisent autour de trois dimensions principales : la position dans les hiérarchies scientifiques officielles ou plus occultes ; la discipline d'appartenance ; les responsabilités institutionnelles locales.

Le prestige scientifique est le produit complexe des réalisations personnelles, de la spécialité et de l'insertion dans les réseaux nationaux et internationaux qui structurent la communauté scientifique. Il intervient à divers niveaux, dont la mobilisation des réseaux de pairs : le conflit autour de la candidature de J.P. Serres à Nancy illustre l'importance de cette dimension. Si ce dernier n'avait pas été considéré par ses collègues comme le meilleur mathématicien de sa génération, le conflit n'aurait pas dépassé un niveau local.

La discipline tend à se définir comme une communauté dont le territoire scientifique fait l'objet d'enjeux importants et de négociations perpétuelles. Chaque enseignant est à la fois l'un des membre de la communauté, occupant une place spécifique au sein des systèmes d'opposition et de solidarité de celle-ci, et son représentant dans le système local. Ce dernier aspect l'investit d'une certaine façon d'une responsabilité institutionnelle puisqu'il dirige en général une équipe et représente souvent à lui seul sa spécialité, qu'il doit défendre lors des attributions de ressources.

Les responsabilités institutionnelles, qu'il s'agisse de la direction d'une équipe ou d'un institut de formation, induisent à la fois des espaces d'action et des devoirs : il faut bien faire fonctionner le dispositif organisationnel dont on a la responsabilité, quels que soient les autres enjeux, ce qui oblige parfois à trancher entre la défense de la discipline et les intérêts de l'institution.

Dans un tel contexte, le conflit de Nancy révèle les résistances que pouvait rencontrer dans les facultés de l'époque l'émergence d'une discipline réputée "appliquée". Ces résistances s'expliquent à la fois par un effet alors assez général de dévalorisation de la recherche appliquée et par jeu des rapports internes aux systèmes scientifiques locaux.

Le faible prestige du calcul numérique au sein des mathématiques ${ }^{28}$ s'inscrit dans un contexte plus général de relégation des sciences les plus appliquées au bas des hiérarchies scientifiques françaises. Ce point, qui renvoie à l'histoire et aux structures profondes du systèmes scientifique français, mériterait à lui seul une analyse détaillée que nous ne pouvons développer ici, mais il constitue un incontournable élément explicatif de la passivité ou des réactions négatives des mathématiciens face à l'émergence des spécialités appliquées. Dans le cas des facultés qui ont ignoré la recherche en informatique durant la période des débuts et qui ont tenté de rattraper leur retard par la suite (cas des logiques exogènes décrites plus haut), c'est plutôt la passivité qui domine : il n'y a pas forcément d'opposition explicite, mais la question du développement du calcul numérique, puis de l'informatique, est soit ignorée (Lyon, Marseille), soit éludée (Bordeaux, Montpellier). Lorsqu'une initiative se fait jour et touche des enjeux importants, la réaction est en général hostile : conflit ouvert à Nancy; difficultés rencontrées par J. Kuntzmann à Grenoble: "L'attitude à notre égard des mathématiciens (purs) a été toute différente (de celle des physiciens). Leur désir essentiel était de préserver leur pureté de toute contamination." (Kuntzmann, 1992, p.15). "Il y avait une rivalité avec les mathématiciens purs, qui craignaient que nous ne prenions leurs places [...] Quand on a construit les bâtiments du campus, nous avons été les premiers installés parce que nous manquions de place. On nous a construit un bâtiment plus grand que celui des maths pures parce qu'il fallait loger les machines" (J. Kuntzmann, entretien, 1990).

L'opposition aux mathématiques appliquées n'est pas le seul fait des mathématiciens "purs", elle résulte aussi des compétitions locales entre disciplines. Dans un jeu à somme finie ${ }^{29}$, un domaine se développe forcément au

\footnotetext{
28 L'informatique se trouvera confrontée à l'opposition des mathématiciens qui lui dénieront longtemps le statut de discipline (Mounier-Kuhn, 1987).

$29 \mathrm{Si}$ dans un jeu à somme nulle, le gain de certains implique une perte pour d'autres, tout le monde peut gagner dans un jeu à somme finie (positive) mais si certains gagnent beaucoup, d'autres ont nécessairement des gains limités ou des pertes. Dans le cas qui nous occupe, la croissance des universités au cours des années soixante se traduit par un jeu de ce type. Par ailleurs, au cours des années cinquente, les acteurs ne prévoyaient pas tous la croissance qui allait venir et pouvaient ajuster leurs stratégies sur la base d'un jeu à somme nulle, défendant avec d'autant plus d'âpreté leur discipline. Enfin, il y a la question des hiérarchies générales ou locales : si le développement d'une discipline ou spécialité ne nuit pas nécessairement à celui des autres, il peut induire une modification des hiérarchies en vigueur.
} 
détriment de la croissance des autres. La réaction des disciplines voisines des mathématiques appliquées relève de cette logique : tant que le calcul numérique, puis l'informatique, se cantonnent à du service (centres de calcul) et à l'enseignement des techniques (programmation), il n'y a pas d'opposition importante. Leur constitution en tant que domaine scientifique à part entière, par le recrutement d'enseignants de haut rang, est en revanche une source de conflits. Si les écoles d'ingénieurs, essentiellement dirigées par des physiciens, ont joué un rôle initial décisif dans le recrutement de mathématiciens appliqués à Grenoble, Toulouse et Nancy, les mêmes physiciens n'ont pas toujours vu ensuite d'un bon œil le développement d'un domaine scientifique nouveau au sein de la faculté : "Les jeunes loups de la physique [...] veulent être les premiers par le prestige et croître sans limites. Une initiative de taille modeste leur plaît, car elle montre la vitalité de Grenoble. Mais si elle prend trop d'amplitude, elle leur paraît une rivale" (Kuntzmann, 1992, p.15). Cela explique aussi peut-être en partie l'essor brisé de l'informatique à Strasbourg où rien ne contrebalançait la logique protectionniste des autres disciplines, qui aboutit au départ du seul "numéricien" local.

Les blocages disciplinaires passifs ou actifs constituent une situation défavorable au développement des mathématiques appliquées. Les cas où celles-ci, le calcul numérique en particulier, arrivent à émerger sont finalement des exceptions dans lesquels ces blocages sont levés sous l'effet de deux logiques : une logique volontariste expliquée par une situation singulière (recomposition de la faculté et politique menée par G. Poitou à Lille, effet "capitale" à Paris) ou une logique institutionnelle spécifique comme celle qui prévaut au sein des systèmes scientifiques de Grenoble, Nancy et Toulouse. Ni Paris ni Lille ne sont "pionniers entre les pionniers" : Grenoble et Toulouse sont déjà largement engagés dans l'enseignement et la recherche en calcul numérique lorsque les autorités nationales décident d'impulser cette spécialité à Paris ; d'autres sites ont commencé à se préoccuper de calcul lorsque les jeunes mathématiciens lillois choisissent de mettre en place des cours dans ce domaine et de s'équiper de calculateurs. On peut donc penser que l'exemple des sites les plus précoces et tout particulièrement celui de Grenoble n'est pas étranger à ces décisions.

La configuration du système scientifique local qui caractérise les trois sites pionniers a donc une grande importance puisqu'elle explique en partie le développement de l'ensemble de l'informatique universitaire française. Cette configuration a pour effet de placer à un moment donné au pemier plan l'impératif de l'enseignement des mathématiques appliquées et d'introduire dans le système de recrutement (Nancy) ou d'orientation scientifique des enseignants déjà présents (Grenoble) une rupture par rapport à la hiérarchie qui prévaut au sein des mathématiques, cette hiérarchie étant contournée par le recrutement d'un physicien à Toulouse. Dans les trois cas, la présence des écoles explique les initiatives visant à développer les mathématiques appliquées, et/ou l'aboutissement de ces initiatives, rendu possible par le poids des écoles dans la configuration interne des Facultés. Le cas de Rennes-Nantes aurait pu suivre cette logique puisque c'est d'une école d'ingénieurs que sont venues les initiatives en faveur du calcul numérique et de l'informatique ; mais la création de la faculté de Nantes, assortie de la nomination du spécialiste du calcul à une responsabilité administrative, a fait basculer le processus vers une autre logique.

La présence des écoles d'ingénieurs en électricité constitue la cause du développement des mathématiques appliquées puis de l'informatique dans deux des trois facultés citées et un contexte favorable à sa croissance dans la troisième (Toulouse). Comme cette configuration n'existe nulle part ailleurs à cette époque, elle apparaît comme une condition suffisante du développement de l'informatique. Le problème de la différenciation des pôles scientifiques se trouve alors à nouveau renvoyé en amont : pourquoi y avait-il à Grenoble, Toulouse et Nancy et non ailleurs des ENSI dans le domaine de l'électricité ?

\section{4 - Les effets à long terme de la création des instituts techniques des facultés des sciences}

Comprendre la configuration particulière des systèmes scientifiques de Toulouse, Grenoble et Nancy nécessite un rapide retour au début du siècle et aux grandes réformes universitaires des années 1876-1897.

Pour une analyse détaillée de ces réformes, on peut se reporter aux travaux de Grelon (1989), Karady (1986), Paul (1980) ou Weisz (1977). Rappelons simplement leur objectif : créer un nombre limité de grandes universités, comparables aux universités allemandes et susceptibles d'améliorer la situation de la recherche française, jugée particulièrement alarmante. Le gouvernement, ne disposant ni des moyens ni d'un consensus politique suffisants pour imposer la création de cinq ou six grandes universités, dut se contenter de réformer l'organisation des enseignements, de créer des chaires d'enseignement et des bourses pour les étudiants, et surtout de donner beaucoup plus d'autonomie aux facultés en les autorisant à faire appel à des financements locaux, privés ou publics, et à générer de nouveaux enseignements.

Dans une situation caractérisée par le relatif retrait de l'autorité nationale et par l'ouverture de l'enseignement supérieur aux initiatives locales, la compétition ouverte entre les villes universitaires se traduit par une 
différenciation profonde. Les efforts consentis par les collectivités locales en faveur de leurs universités sont à la fois très importants et variables, du point de vue tant quantitatif (sommes investies) que qualitatif (orientations, création de nouveaux enseignements). Les demandes des industriels et les espoirs économiques des responsables locaux aboutissent à l'ouverture de nouveaux enseignements appliqués à l'industrie (Grelon, 1989). Sont ainsi créés de nouvelles chaires, des cours publics, puis des instituts techniques annexes des facultés des sciences destinés à la fois à développer des recherches et à former des ingénieurs pour les entreprises locales. Les orientations disciplinaires des instituts créés alors peuvent se classer en quatre catégories : la chimie (10 instituts), l'agriculture et l'agro-alimentaire ( 3 instituts auxquels il faut ajouter les instituts d'oenologie et l'école de brasserie), diverses spécialités liées aux milieux industriels locaux (tannerie, papeterie, etc.), et enfin l'électricité avec les instituts d'électrotechnique de Nancy (1901), Grenoble (1901), Lille ${ }^{30}$ (1905) et Toulouse (1907).

Le choix de l'électrotechnique s'explique par les contextes locaux : essor de l'hydroélectricité à Grenoble ; espoir d'un développement fondé sur la "houille blanche" à Toulouse, situation particulière de Nancy dans cette période et présence de puissants mécènes industriels (Grossetti, 1994a).

Ces mêmes instituts, diversifiés (aux enseignements d'électrotechnique se sont ajoutées l'hydraulique et l'électronique) et transformés en Écoles nationales supérieures d'ingénieurs (ENSI), sont ceux que nous avons retrouvés associés au démarrage du calcul numérique et de l'informatique à l'université, ainsi que celui de l'automatique dont les premières équipes universitaires françaises ont éclos dans les laboratoires d'électrotechnique (Grossetti, 1991, 1993).

\section{Conclusion}

En parcourant à rebours le développement de la recherche en informatique dans les universités, nous avons pu isoler un certain nombre de régularités. La première concerne le lien entre calcul numérique et informatique qui se vérifie dans tous les cas. La configuration du système scientifique de l'époque et l'échec des recherches précédentes sur les calculateurs expliquent ce lien. De ce fait, le problème des débuts de l'informatique se déplace vers l'émergence des équipes de recherche en calcul numérique, et plus généralement en mathématiques appliquées. L'analyse des situations locales permet de mettre au jour plusieurs facteurs s'opposant au développement de ces spécialités dans la période considérée : le faible prestige des sciences appliquées en général ; le contexte spécifique des mathématiques alors dominées par le groupe "Bourbaki" aux orientations très fondamentalistes ; les rivalités entre disciplines au sein des facultés des sciences. Chacun de ces points mériterait une étude d'ensemble qui déborde largement la cadre de notre recherche ${ }^{31}$. Face à ce contexte défavorable, il existe une demande sociale pour les mathématiques appliquées, puis pour l'informatique. Au début, il s'agit simplement de la formation au calcul numérique des ingénieurs électriciens, mais très vite il s'agira de toute la pression sociale liée à l'informatique, incomparablement plus forte, avec le Plan Calcul et l'essor considérable de l'industrie dans ce secteur. La configuration institutionnelle associant des écoles d'ingénieurs et une faculté des sciences a permis à cette demande sociale de pénétrer dans le système universitaire, alors assez fermé, et de le faire évoluer, aboutissant dans certains sites à la constitution d'équipes de calcul numérique, puis d'informatique, qui se sont ainsi retrouvées en situation de quasi-monopole pour ces spécialités (y compris face à la capitale durent quelques années). C'est ainsi que les modestes équipes de numériciens, en devenant des laboratoires d'informatique, connaitront un essor coinsidérable, bénéficiant d'une manne régulière de ressources de tous ordres (moyens de calcul, financements, postes) ${ }^{32}$. Ainsi, sur la base de situations précaires, partiellement contingentes, s'est construit une hiérarchie durable des pôles français de recherche en informatique ${ }^{33}$, contribuant à renforcer le potentiel économique des villes qui leurs sont associées ${ }^{34}$.

L'analyse du système scientifique présentée ici vaut pour une courte période seulement : dès la fin des années soixante, l'informatique tend à devenir une discipline indépendante de l'analyse numérique, ou du moins à la déborder largement ; les rapports de force entre mathématiciens "purs" et "appliqués" ont beaucoup évolué par la suite ; la création du département "Sciences Pour l'Ingénieur" au CNRS a permis aux disciplines appliquées de

\footnotetext{
30 L'institut d'électrotechnique de Lille ne pourra se développer faute d'étudiants, la plupart des candidats potentiels étant absorbés par une institution concurrente, l'Institut Industriel du Nord (actuelle Ecole centrale de Lille)

31 Une étude des effets du groupe "Bourbaki" sur les mathématiques françaises serait à cet égard très intéressante.

32 Justifiant ainsi d'un certain point de vue les craintes de leurs adversaires !

33 Selon un tri des équipes de la section 7 du CNRS, Grenoble, Toulouse et Nancy rassemblent $36 \%$ des chercheurs et enseignants-chercheurs des équipes de recherche en informatique, soit $55 \%$ du total des pôles de province.

34 L'informatique est l'une des disciplines qui produit le plus de collaborations entre la recherche publique et l'industrie. Par exemple, $13 \%$ des conventions CIFRE (co-financements de thèses par des entreprises) concernent des équipes d'informatique, ce qui constitue la plus importe contribution avec la chimie (14\%).
} 
trouver une expression institutionnelle ; les ENSI ont pris une plus grande autonomie vis-à-vis des universités après 1968 ; en particulier, celles de Grenoble, Toulouse et Nancy se sont réorganisées en 1970 sous la forme d'Instituts Nationaux Polytechniques disposant de leurs propres enseignants.

Par ailleurs, l'émergence de l'informatique se produit à une époque où les Facultés des Sciences sont peu influencées par leur environnement local : la loi les fait dépendre totalement des institutions nationales, ne leur permet ni de prendre l'initiative de nouveaux diplômes, ni de bénéficier de financements privés ; elles ont relativement peu de liens avec les entreprises, sauf dans le cas de Grenoble (Pestre, 1990). En remontant le temps pour expliquer la présence des écoles d'électricité à Grenoble, Toulouse et Nancy, nous avons rencontré un autre contexte où les environnements locaux pouvaient imprimer profondément leurs marques aux établissements scientifiques selon une gamme de logiques beaucoup plus large. A. Grelon (1989) a jeté les bases d'une analyse de ce qui s'est joué sous la République radicale sur le plan des institutions scientifiques françaises. Il resterait à saisir plus précisément les facteurs de la différenciation des pôles scientifiques dans cette période.

Les débuts de l'informatique dans les universités témoignent donc d'une phase particulière du processus de différenciation des pôles scientifiques français, celle qui après la création des centres académiques (1808 - 1870) et leur différenciation (1870 - 1939), a vu le rattrapage du retard en français dans le domaine des sciences appliquées renforcer les disparités héritées du début du siècle. Les facultés de Province ont joué un rôle décisif pour combler ce retard, prouvant ainsi qu'elles peuvent avoir des capacités d'innovation bien supérieures à celles qu'on leur prête habituellement, grâce à leur insertion dans des systèmes scientifiques locaux associant recherche fondamentale et formations d'ingénieurs. 


\section{RÉFÉRENCES}

BELHOSTE, B., 1989, “Les origines de l'École Polytechnique. Des anciennes écoles d'ingénieurs à l'école centrale des Travaux Publics”, Histoire de l'Éducation, $\mathrm{n}^{\circ} 42$, Mai , pp. 13-53

BEN-DAVID J. et ZLOCKZOWER A., 1962, "Universities and academic systems in modern societies", Arhives européennes de Sociologie, III

BEN-DAVID J., 1968, La recherche fondamentale et les universités, OCDE

BENSOUSSAN A. "De l'IRIA à l'INRIA", Actes du 2e Colloque sur l'Histoire de l'Informatique en France, CNAM, Paris, 1990.

BRETON, P. 1990, Une histoire de l'informatique, La Découverte-Points Sciences

BROCARD M., 1991, La science et les régions, La Documentation française

BURNEY J.-M., 1988, Toulouse et son université, Pum - CNRS

CALLON M. (sous la direction de), 1989, La science et ses réseaux, Genèse et circulation des faits scientifiques, La découverte, Paris

CALLON M., LARÉDO P. et MUSTAR P., 1994, "Panorama de la science française", La Recherche, n²64, Avril, Vol 25

CHATELIN P. (Ed), 1988, Colloque sur l'histoire de l'informatique en France (Actes), Grenoble

CNRS, 1992, "Les relations du CNRS avec les entreprises", Mission des relations avec les entreprises

COLLINS, 1974, "The T.E.A. set : tacit knowledge and scientific networks", Science Studies, nº 4 , pp.165-186

DROUARD A., 1978, Analyse comparative des processus de changement et mouvements de réforme dans l'enseignement supérieur français, Ed CNRS, Atp 25

FOX et WEISZ, 1981 (ed.), The Organization of science and technology in France 1808-1914, Cambridge University Press et Éditions de la Maison des Sciences de l'Homme

GAILLARD G., 1991, (avec la collaboration de C. DETREZ et E. BORENFREUND), “Analyse et schéma de développement des pôles de compétence français en génie des procédés", rapport de mission pour le MRT, $40 \mathrm{p}$.

GRELON A. et TERNIER A., 1986, "Chronologie des ingénieurs (1744-1985)", in GRELON A (sous la direction de), Les ingénieurs de la crise, Ed. Ehess

GRELON A., 1989, "Les universités et la formation des ingénieurs en France (1870-1914)", in Formation emploi, n'27-28

GRELON A., 1987, "La question des besoins en ingénieurs de l'économie française. Essai de repérage historique", Technologies - Idéologies - Pratiques, VI/4,

VII/1, Université de Provence, pp. 3-22

GROSSETTI M., 1990, "Enseignement supérieur et technopole : l'exemple de l'informatique à Toulouse”, Revue Française de Sociologie, XXXI, n³

GROSSETTI M., 1991, "Structuration territoriale des sciences appliquées en France, Étude comparative de Toulouse, Grenoble et Nancy, rapport pour le PIRTTEM-CNRS, $84 \mathrm{p}$.

GROSSETTI M., 1993,, "Les débuts de l'informatique et de l'automatique dans les universités de Grenoble, Toulouse et Nancy", Communication pour le 3e Colloque d'Histoire de l'Informatique, Sophia-Antipolis, 13-15 Octobre 1993

GROSSETTI M., 1994a, "Villes et institutions scientifiques - Genèse des pôles scientifiques français, Annales de la Recherche Urbaine, ${ }^{\circ} 62-63$, Juin

GROSSETTI M., 1994b, "Sciences de la parole : genèse d'une communauté scientifique en France" Communication pour les Journées d'Études sur la Parole, Lannion, 1er au 3 Juin 1994

LABORIE M., "L'informatique à l'Université de Toulouse" Actes du 2e Colloque sur l'Histoire de l'Informatique en France, CNAM, Paris, 1990.

KARADY. V., "De Napoléon à Duruy : les origines et la naissance de l'université contemporaine" et "Les universités et la troisième république" in (Sous la direction de J. Vergé), Histoire des Universités en France, Privat, Toulouse, 1986.

KUNTZMANN J., 1992, "Naissance et jeunesse de l'IMAG", IMAG

KNORR-CETINA K. et MULKAY M. (eds), 1983, Science observed. Perspectives on the social study of science, Sage, Londres

HOFFSAËS C., 1988, "L'AFCET et les sociétés qui l'ont constituée", Colloque sur l'histoire de l'informatique en France (Actes), Grenoble, 1988

LATOUR B. ET WOOLGAR S., 1988 (1979), La vie de Laboratoire. La production des faits scientifiques, La Découverte, Paris

LATOUR B., 1989, La science en action, La découverte, Paris

LATOUR B., 1989, "Pasteur et Pouchet : hétérogenèse de l'histoire des sciences", dans M. Serres, Éléments d'histoire des sciences, Bordas, Paris

LECUYER B.-P., 1978, "Bilan et perspective le la sociologiue de la science dans les pays occidentaux", Archives Européennes de sociologie, XI X

LEVY P., 1989, "L'invention de l'ordinateur", in M. Serres (ed), Éléments d'histoire des sciences, Bordas

LYNCH M., 1982, "Technical work and critical enquiry : investigations in sa scientific laboratory", Social Studies of Science, $\mathrm{n}^{\circ} 12, \mathrm{pp} .499-533$

LYNCH M., 1985, Art and Artifact in laboratory Science : A Study of Shop Work and Shop Talk in a Research Laboratory, Routhledge and Kegan Paul, Londres

MAILFERT A., 1991, Recherche et territoire, La documentation française

MERTON R.-K., 1965, Eléments de théorie et de méthode sociologique, Paris, Plon (Première édition 1953)

MERTON R.-K., 1973 (ed), The Sociology of Science, Chicago / Londres, University of Chicago Press

MOUNIER-KUHN P.-E., 1987 Le Comité national et l'émergence de nouvelles disciplines au CNRS : le cas de l'informatique 1946-1976. Mémoire de DEA,

Centre Science, Technologie et Société, CNAM, Paris.

MOUNIER-KUHN P.-E., 1988 "L'Institut Blaise-Pascal du CNRS (1946-1969)" Colloque sur l'histoire de l'informatique en France. Grenoble.

MOUNIER-KUHN P.-E., 1989,“The Institut Blaise-Pascal (1946-1969) - From Couffignal's Machine to Artificial Intelligence” Annals of the History of Computing vol. 11/4.

MOUNIER-KUHN P.-E., 1990, "Genèse de l'informatique en France (1945-1965). Diffusion de l'innovation et transfert de technologie”, Culture Technique.

MOUNIER-KUHN P.-E., 1994 "La recherche publique française et les premiers ordinateurs : un échec lourd de conséquences (1939-1959)", Actes des Journées d'étude sur la Parole, CNET Lannion, pp.23-29.

PAUL H. W., "Apollo courts the Vulcains - The applied science institutes in nineteenth century french science faculties" in C. Fox et G. Weisz, The organization of Science and technology in France, 1808-1914, MSH, 1980

PESTRE D., 1990, "Louis Néel, le magnétisme et Grenoble. Récit de la création d'un empire physicien dans la province française, 1940-1965", Cahiers pour l'histoire du CNRS, n8, 1990, Éditions du CNRS

PICARD J.-F., La république des savants. La recherche française et le C.N.R.S., Flammarion, 1990

PLENET C., "Mémoire et légende des informaticiens de Grenoble", mémoire de maîtrise sous la direction de J.-P. Bozonnet, Université de Grenoble II, UFR de Sociologie, 1990

PROST A., Histoire de l'enseignement en France, 1800 - 1967, Paris, A. Colin, 1968

RAMUNNI G., "Louis Couffignal (1902-1966) : un pionnier de l'informatique en France ?" in P. Chatelain (ed.), Colloque sur l'histoire de l'informatique en France (Actes), Grenoble, 1988

RAYMOND F.-H., "Une aventure qui se termine mal : la SEA, in P. Chatelain ed, Colloque sur l'Histoire de l'informatique en France, 1988

RUSSO F., 1977, "Science et technique" in B. Gille, Histoire des techniques, La Pléiade

SFEZ L, 1976, Critique de la décision, FNSP

SHINN T., "Des sciences industrielles aux sciences fondamentales - La mutation de l'École supérieure de physique et de chimie (1882 - 1970)", Revue Française de Sociologie, XXII, 1981, pp. 167-182

THEPOT A., "Les institutions scientifiques et techniques au XIXe siècle", in Histoire de l'éducation, №18, avril 1983.

VERGER J et VULLIEZ CH, "Naissance de l'université", in (Sous la direction de J. Vergé), Histoire des Universités en France, Privat, Toulouse, 1986.

WEISZ G, "Le corps professoral de l'enseignement supérieur et l'idéologie de la réforme universitaire en France, 1860 - 1885", Revue Française de Sociologie, $\mathrm{n}^{\circ} 18,1977$

ZWERLING C. "The emergence of the École Normale Supérieure as a centre of scientific education in the nineteenth century", in R. Fox et C Weisz, The organization of Science and technology in France, 1808-1914, MSH, 1980 